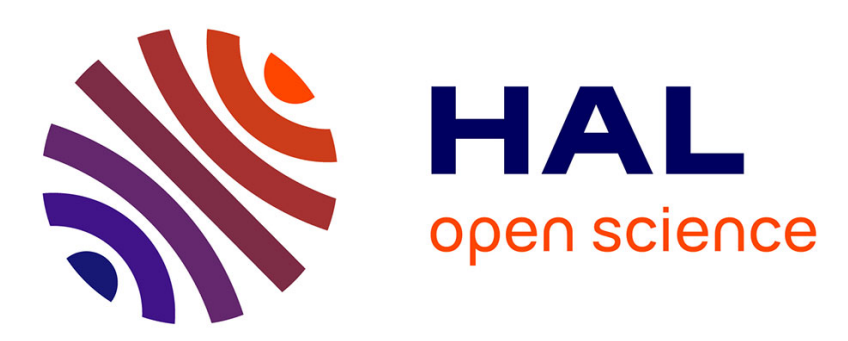

\title{
Mastered dispersion of material resonators: Broad corrugated waveguides working under the Littrow regime
}

\author{
Henri Benisty, Nikolay Piskunov
}

\section{- To cite this version:}

Henri Benisty, Nikolay Piskunov. Mastered dispersion of material resonators: Broad corrugated waveguides working under the Littrow regime. Applied Physics Letters, 2013, 102, pp.151107. 10.1063/1.4802253 . hal-00857663

\section{HAL Id: hal-00857663 \\ https://hal-iogs.archives-ouvertes.fr/hal-00857663}

Submitted on 18 Nov 2015

HAL is a multi-disciplinary open access archive for the deposit and dissemination of scientific research documents, whether they are published or not. The documents may come from teaching and research institutions in France or abroad, or from public or private research centers.
L'archive ouverte pluridisciplinaire HAL, est destinée au dépôt et à la diffusion de documents scientifiques de niveau recherche, publiés ou non, émanant des établissements d'enseignement et de recherche français ou étrangers, des laboratoires publics ou privés.

\section{(c) (1) $\$$}

Distributed under a Creative Commons Attribution - NonCommerciall 4.0 International 


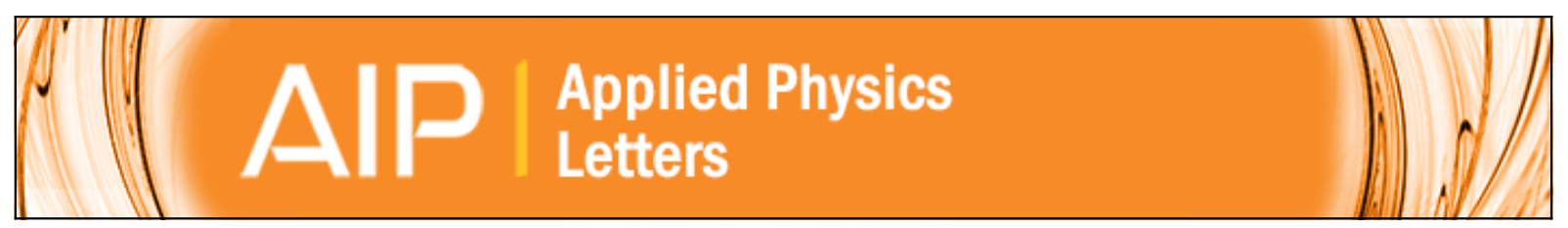

Mastered dispersion of material resonators: Broad corrugated waveguides working under the Littrow regime

H. Benisty and N. Piskunov

Citation: Applied Physics Letters 102, 151107 (2013); doi: 10.1063/1.4802253

View online: http://dx.doi.org/10.1063/1.4802253

View Table of Contents: http://scitation.aip.org/content/aip/journal/apl/102/15?ver=pdfcov

Published by the AIP Publishing

\section{Articles you may be interested in}

Mid-infrared intersubband polaritons in dispersive metal-insulator-metal resonators

Appl. Phys. Lett. 105, 081105 (2014); 10.1063/1.4893730

Mechanism of azimuthal mode selection in two-dimensional coaxial Bragg resonators

J. Appl. Phys. 105, 124519 (2009); 10.1063/1.3143019

Coupled resonator optical waveguides based on silicon-on-insulator photonic wires

Appl. Phys. Lett. 89, 041122 (2006); 10.1063/1.2234721

Zero dispersion at small group velocities in photonic crystal waveguides

Appl. Phys. Lett. 85, 4866 (2004); 10.1063/1.1815066

Heavy photon dispersions in photonic crystal waveguides

Appl. Phys. Lett. 77, 178 (2000); 10.1063/1.126916

\section{AIP $\left.\right|_{\text {APL Photonics }}$}

APL Photonics is pleased to announce Benjamin Eggleton as its Editor-in-Chief

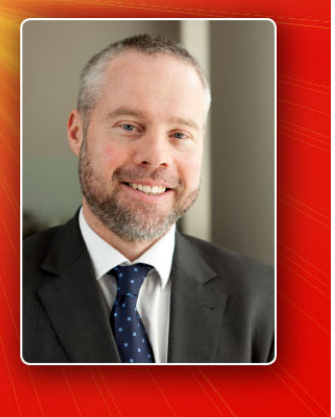




\title{
Mastered dispersion of material resonators: Broad corrugated waveguides working under the Littrow regime
}

\author{
H. Benisty and N. Piskunov \\ Laboratoire Charles Fabry, Institut d'Optique, CNRS, Univ Paris Sud, 2, Avenue Augustin Fresnel, \\ 91127 Palaiseau cedex, France
}

(Received 13 February 2013; accepted 4 April 2013; published online 17 April 2013)

\begin{abstract}
An anomalous dispersion for modes of a material resonator is highly desired to form frequency combs. A resonator free-spectral-range (FSR) controlled by shape so as to increase with frequency $\omega / 2 \pi$ compensates the normal index dispersion $\partial n / \partial \omega>0$, producing evenly spaced resonances. Only special shapes achieve this scope. We show here that broad periodic corrugated waveguides working at Littrow regime feature such an increasing trend $\partial \mathrm{FSR} / \partial \omega>0$. We outline experimentally this trend on silicon-on-insulator devices designed for $45^{\circ}$ Littrow operation. We predict dispersion-free silicon-based designs across the $1.4-4.0 \mu \mathrm{m}$ mid-infrared range. (C) 2013 AIP Publishing LLC [http://dx.doi.org/10.1063/1.4802253]
\end{abstract}

Accuracy of frequency metrology is hugely benefiting from the use of frequency combs. The possibility has emerged to get such stable combs not by a pulsed laser-further fed to a supercontinuum generation device-but directly from the spontaneous instability of a cw beam in a material resonator, feeding a broad bunch of modes through nearresonant $\chi^{(3)}$ nonlinear mechanisms. However, triggering the instability requires that at least a few seed modes align their free spectral range (FSR), thus inducing phase-matched sum and difference frequencies. ${ }^{1}$ This research started around studies of whispering-gallery modes in microspheres. ${ }^{2,3}$ It has further actively targeted resonators whose FSR is "liberated from dispersion," for instance made of a calcite truncated ellipsoid ${ }^{4}$ or from carefully designed $\mathrm{Si}_{3} \mathrm{~N}_{4}$ rings. 5 " Such designs impose stringent relations between materials and shape, leaving very few free parameters. Silica microtores appear to have similar requirements. ${ }^{7}$ Recently, related aspects, such as broadband polarization independence of dispersion in silicon waveguides ${ }^{8}$ and capillary-type cylindrical resonator modal management, ${ }^{9}$ were also tackled along interesting avenues. Generally, constraining optical features, such as the FSR absolute value or modal characteristics such as confinement factors, penetration or effective index differences, are not desirable for further engineering of such systems, hence the interest for options that lessen such constraints.

Here, we show that periodic broad corrugated waveguides $(\mathrm{BCW})$ have a potential for a "dispersion-liberating" or said more conventionally, a dispersion-compensating engineering, with added versatility. We previously studied these highly multimode structures (i) for their "Littrow lasing" feature ${ }^{10}$ and (ii) for their ability to manifest the phenomena of "multiple slow light" or "critical coupling."11-14 These slow modes arise at the crossings of equidistant branches of two dispersion manifolds of opposite slopes. ${ }^{15}$ Coupling of modes with proper amounts of transverse and longitudinal momentum $^{4}$ thus appears as a generic enabler for dispersion compensation strategies. The control of special electromagnetic features in highly multimode waveguides could also benefit from knowledge of the quasi-optics community. ${ }^{16}$
In Fig. 1, we depict a generic "Littrow resonator" much as those of Ref. 15 of period $a$ and width $w$ but for the unfolding along the dashed-dotted line, done in order to remain more generic. Its resonant frequencies originate from stationary points of the modes $\omega=\omega\left(k_{x}\right)$ of the infinite BCW. Here, nearly flat bands essentially result from the crossing, at or around the longitudinal wavevector $k_{x}=\pi / a$, of the elementary manifold of the broad waveguide (Fig. $1(\mathrm{~b})$ ), with its folded counterpart $\left(k_{x} \rightarrow 2 \pi / a-k_{x}\right)$. The manifold branches are simply in the hard-wall limit

$$
\omega_{\mathrm{p}} \approx\left(\mathrm{c} / n_{\mathrm{z}}\right)\left[(\mathrm{p} \pi / w)^{2}+\left(k_{x}\right)^{2}\right]^{1 / 2},
$$

$n_{\mathrm{z}}=n_{\mathrm{z}}(\omega)$ being the underlying slab effective index. The Littrow "retrodiffraction" condition, which also reads $k_{\mathrm{x}}^{\prime}=-k_{x}[2 \pi / a]$, is thus obviously obeyed at $k_{x}=\pi / a{ }^{10,15}$ Whatever the details of coupling among the crossing manifolds, the resulting stationary frequencies (weakly curved dotted hyperbola of Fig. 1(b)) are known to be constrained by these crossings, and thus their FSR essentially follows the "Littrowian" FSR, FSR $_{\mathrm{L}}=\Delta \omega / 2 \pi$, which derives from the intervals of the series $\omega_{\mathrm{L}, \mathrm{p}}=\left(\mathrm{c} / n_{\mathrm{z}}\right)\left[(\mathrm{p} \pi / w)^{2}+(\pi / a)^{2}\right]^{1 / 2}$. It is clear that $\mathrm{FSR}_{\mathrm{L}}$ grows from a small value near the light line $\left(\omega_{\mathrm{p}}=k_{x} \mathrm{c} / n_{z}\right)$, i.e., when $p \rightarrow 1$, to the asymptotic Fabry-Perot (FP) value $\mathrm{FSR}_{\mathrm{FP}}=\left(\mathrm{c} / 2 n_{z} w\right)$, since $2 \pi \nu_{\mathrm{p}}=\omega_{\mathrm{p}} \sim\left(\mathrm{c} / n_{z}\right)(p \pi / w)$ in this regime $p \rightarrow \infty$. This is the essential mechanism of negative dispersion that we exploit here. To our knowledge, this basic implementation of negative dispersion has not been reported for resonators.

Our first step is the experimental demonstration that the BCW-based resonators for $\theta_{\mathrm{L}}=45^{\circ}$ Littrow operation, similar to those of Ref. 15, do exhibit this property. These are siliconon-insulator (SoI) samples processed by EpixFab, ${ }^{17}$ whose slab effective index $n_{z}=2.83$ for TE polarization at $\lambda=1.55 \mu \mathrm{m}$ is well characterized. These finite BCW have sawtooth lateral corrugation, of height $h$, on one side only, which amounts to fold Fig. 1(a) onto the dashed-dotted line. We adapt the definitions of $w$ and $\mathrm{p}$ for this issue to be transparent. We believe that the appearance of our Littrow resonator is more compelling and helps better the intuition with the 


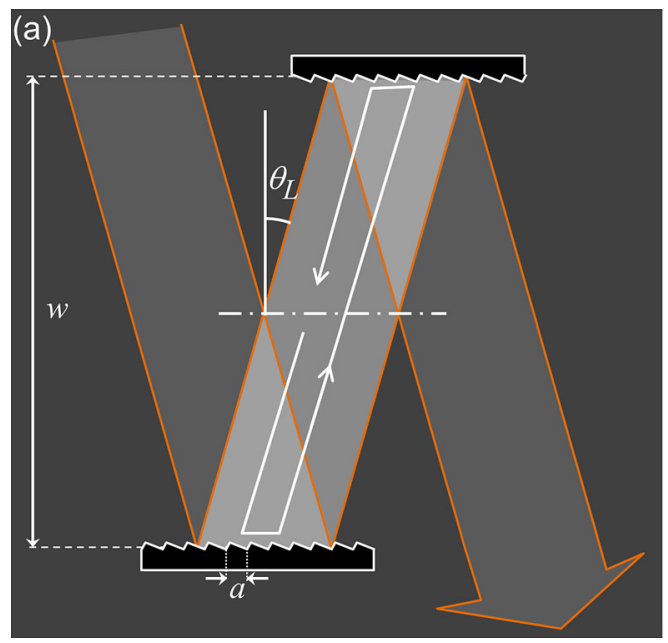

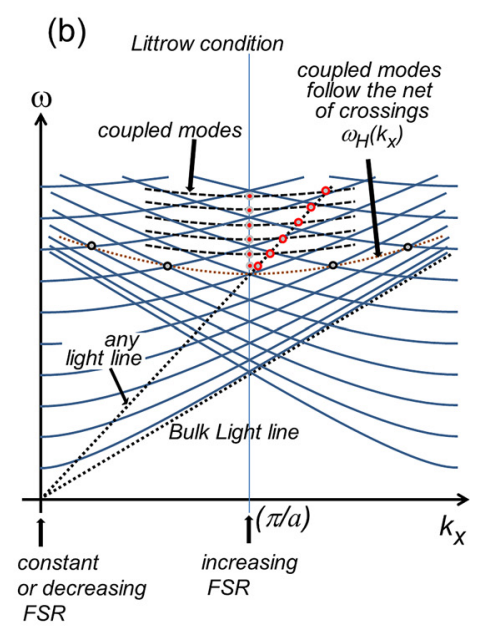

FIG. 1. (a) Scheme of a resonator formed by a broad waveguide section in the Littrow regime. The dashed-dotted line is a mirror plane. The actual devices are folded on this plane and have a single grating (Ref. 15). (b) Dispersion of a broad waveguide (solid line) with the effect of periodicity (folding at $k_{x}=\pi / a$ ). Coupled modes are dashed lines, and follow, modulo the splitting, the net of crossings of basic modes [dotted hyperbola $\omega_{\mathrm{H}}\left(k_{x}\right)$ and black circles]. They have increasing FSR at $k_{x}=\pi / a$ (dots). The excitation along a light line, associated to a given angle in practice, hits the indicated points of the coupled modes in a way that further increases the FSR dispersion. "unfolded" choice of Fig. 1. The corrugation is of a large aspect ratio $h / a$, namely $h / a \sim 2.5$. The width $w$ of the BCWs corresponds to a resonator order $m=75$. ${ }^{15}$ We have $m=2 p$, a factor of 2 coming from the degeneracy lifting of the crossing (another factor would come from operating near $\theta_{\mathrm{L}}=45^{\circ}$, $k_{x} \equiv \pi / a=\mathrm{p} \pi / w \equiv k_{y}$, but it is compensated by the unfolding, doubling $w$ and $m$ ). We contact them by broad access guides tilted at $\theta_{\mathrm{L}}=45^{\circ}$. We then observe the resonances thanks to grating couplers and adequate optics to inject a tunable laser. We monitor the output power $\mathrm{P}(\lambda),(\lambda=2 \pi \mathrm{c} / \omega)$, and analyse its peaks. The BCW length is denoted by $\mathrm{T} q, q$ being an even integer, since ray optics grants that tiling $q$ triangles allows developing $(q-2) / 2$ coupled resonances in the actual folded resonator. ${ }^{15} \mathrm{~T} 4$ devices with a single resonance are most exemplary of our proposal, but devices with several split resonances like T6 reported here are also of interest to accommodate richer dispersion features.

The devices analysed here have better couplers than those used in Ref. 15, but are fully similar in other respects. Thus, we could get a spectrally extended set of resonances, and unambiguously assess the increasing FSR trend, given the well-known modal + chromatic dispersion of $n_{z}(\omega), \partial n_{z}(\omega) / \partial \omega>0$.

In Fig. 2(b), we show the spectra of a representative T4 device, nominally $m=75$ and $h / a=2.5$. We treated the spectrum to extract the FSR as neutrally as possible. We convert $\mathrm{P}(\lambda)$ to $\mathrm{P}(\omega)$, compensate the spectrum for the bell-shaped grating coupler efficiency, and use $\mathrm{P}(\omega)^{2}$ to minimize a first time the background influence. We then form the sliding correlation function $\mathrm{C}(\omega, \Delta \omega) \equiv \int \mathrm{P}\left(\omega^{\prime}-\Delta \omega / 2\right)^{2} \mathrm{P}\left(\omega^{\prime}+\Delta \omega / 2\right)^{2} \mathrm{~d} \omega^{\prime}$, restricting $\omega^{\prime}$ to a window of width $\sim 1.3 \mathrm{THz}$ and sliding center $\omega$. We then used the centroid $\Delta \omega_{\mathrm{c}}(\omega)$ of $\mathrm{C}(\omega, \Delta \omega)^{\mathrm{s}}$ with $s=4$ to further minimize the influence of tails in $\mathrm{C}$. ${ }^{18}$ The result is robust against the choice of the window, we only weighted on Fig. 2(c) the dot size of these centroids by $\mathrm{P}(\omega)$ to better outline the relevant flat regions of $\Delta \omega_{c}(\omega)$. The resulting series is clearly ascending, showing a strong anomalous dispersion $\partial F S R / \partial \omega>0$, the last point being off the rest (see Refs. 10 and 19 with more spectral features of "Littrow resonators"). As for the T6 spectrum (Fig. 2(a), displaying split peaks), its FSR analysis was performed directly from the data. The pattern is not monotonous, but overall similar to T4.

We account only for a fraction of this behaviour by the naive Littrow model combined with the known SoI dispersion
$n_{\mathrm{z}}=n_{\mathrm{z}}\left(\omega_{\mathrm{L}}\right)$ (we use the dispersive indices of the literature and the nominal silicon thickness $217 \mathrm{~nm}$ from our slab index). The total dispersion, if the resonators correspond exactly to the band edge $k_{x}=\pi / a$, is found by solving $\omega_{\mathrm{L}, \mathrm{p}}=\left(\mathrm{c} / n_{\mathrm{z}}\left(\omega_{\mathrm{L}}\right)\right)\left[(\mathrm{p} \pi / w)^{2}+(\pi / a)^{2}\right]^{1 / 2}$ for $\omega_{\mathrm{L}}$, and by forming the intervals $\mathrm{FSR}_{\mathrm{p}}$. In practice, we invert the formula to
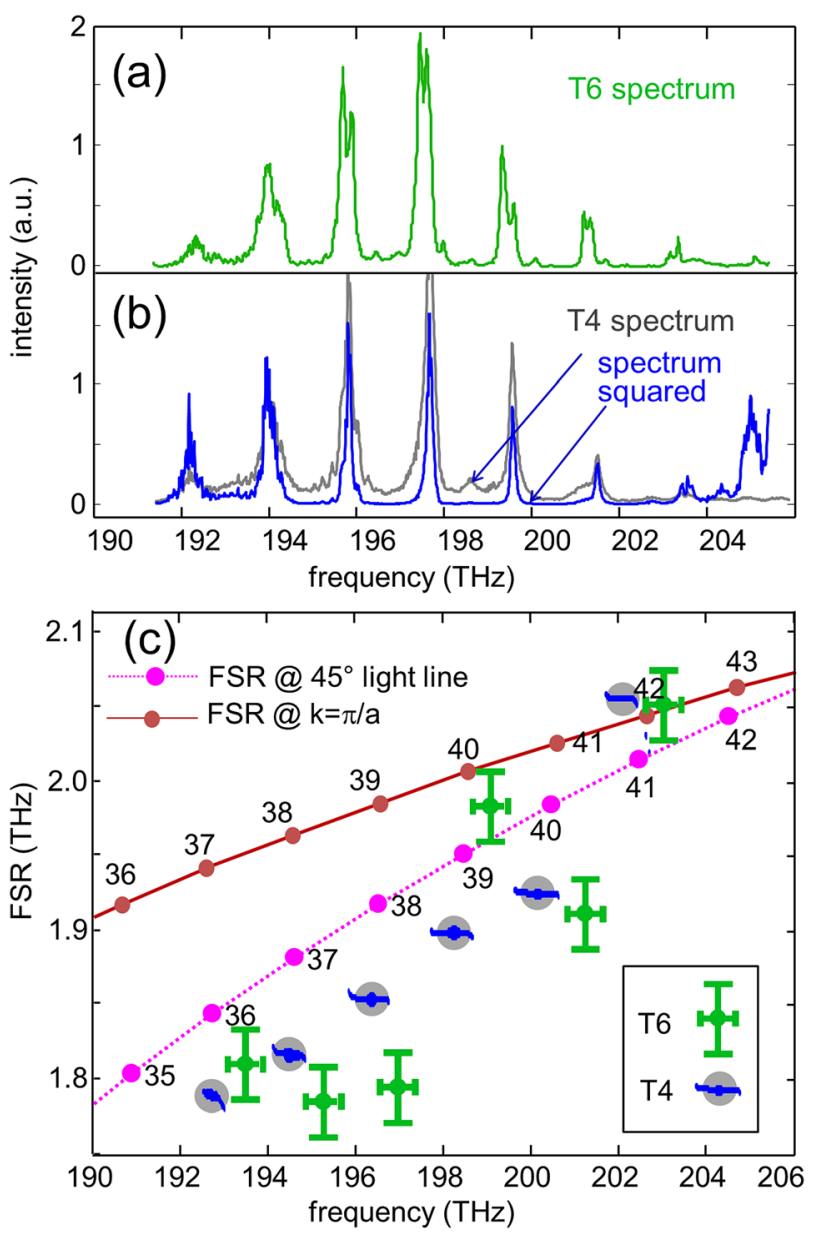

FIG. 2. (a) Raw collected spectrum of a T6 device, twice the length of Fig. 1(a). (b) Raw spectrum of a T4 device, and same spectrum squared and with overall compensated profile, suited for automated FSR determination. (c) Calculated FSR for the $k_{x}=\pi / a$ case (dots and full line) and the $45^{\circ}$ light line case (dots and dotted line) and for the experimental T4 data (grey dots and correlation account by the local centroid of $\Delta \omega_{\mathrm{c}}(\omega)$ of $\mathrm{C}(\omega, \Delta \omega)^{\mathrm{s}}$ with $s=4$ ) and T6 (large crosses). 
calculate fractional orders $\mathrm{p}=\mathrm{p}\left(\omega_{\mathrm{L}}\right)$ on a fine mesh of $\omega$ and interpolate the result at integer orders. The only free parameter is the width, since we simplify and neglect any detail of the actual grating and coupled modes in this way. The advantage exploited later below is the capability to scan a large parameter space. Using a reasonable width of $w=14.6 \mu \mathrm{m}$ ( $\sim$ twice the actual width, the actual devices of Ref. 15 are folded), we get the thick line, whose slope $(\partial \mathrm{FSR} / \partial \omega)_{\text {theo }}$ is nearly one half of $(\partial \mathrm{FSR} / \partial \omega)_{\exp }$.

To account more faithfully for the observed dispersion, we have to take into account the geometry used to optically access these devices. There could be other ways, (see below) but in our current way at fixed angle, the projection $k_{x}$ is proportional to $\omega$, forming a specific "light-line" $k_{x}=\sin \theta n_{z} \omega /$ c. This is illustrated in Fig. 2(b). Due to the residual curvature of the coupled modes, the FSR tends to grow faster toward the upper right, and slower toward the lower left, thus increasing the perceived dispersion. This behavior points out that our resonators still borrow features of a waveguide: as long as no curvature or localizing feature is added to them, they are a section of a perfect periodic waveguide. To get the math of this effect is slightly more cumbersome. The reader can establish by various means ${ }^{20}$ the generic expression of "hyperbola" $\omega_{\mathrm{H}}\left(k_{x}\right)$ based on the net of crossing, Fig. 1(a), which forms the relevant frame for the coupled modes dispersion

$\omega_{\mathrm{H}}\left(k_{x}\right)=\left(\mathrm{c} / n_{\mathrm{z}}\right)\left[(\pi / a)^{2}+(\mathrm{p} \pi / w)^{2}+\Delta k_{x}^{2}\left(1+\mathrm{L}^{2} / \mathrm{p}^{2} a^{2}\right)\right]^{1 / 2}$

with $\Delta k_{x}=k_{x}-\pi / a$. Then, injecting a fixed light-line relation $\Delta k_{x} \propto\left(\omega-\omega_{\mathrm{o}}\right)$, algebra gives $p^{2}$ as the solution of a second degree equation. We then follow the same procedure as above, calculating $p(\omega)$ continuously and interpolating at integers to find resonances. Fig. 2(b) shows that this effect reconciles quite well the experimental and modelled slope. There is still a shift of the order of $2 \mathrm{THz}$ to perfectly fit, but this is not our scope here, we note only that the phase response of the grating would have to be known.

As for the more erratic values of FSR for the longer T6 device, we attribute their shifts to the marginally wavy nature of dispersion around minima of Fig. 1(b) (see also Ref. 15), which plays a bigger role for longer devices (more k-resolution for a more extended mode), and could also favor a more complex interplay with excitation geometry.

Having assessed the strong anomalous dispersion in those specific $45^{\circ}$ Littrow resonators, we now consider the possibility to get exact dispersion compensation in a general Littrow resonator that counteracts the typical "weak" normal dispersion $n_{z}(\omega)$ of slab waveguides used in integrated silicon photonics. The general Littrow resonator design bases its resonances on the crossings at $\omega_{\mathrm{p}}=\left(\mathrm{c} / n_{\mathrm{z}}\right)\left[(\mathrm{p} \pi / w)^{2}+(\pi /\right.$ $\left.a)^{2}\right]^{1 / 2}$. We can indeed take this simpler situation corresponding to the flatter case in Fig. 2(b) if we assume that we excite the resonator at normal incidence through or upon the mirror with an ideal double period grating, so that the diffracted value of $k_{x}(\equiv 2 \pi / 2 a)$ is fixed at $\pi / a$ at any wavelength.

As we had a strong overcompensation of the $\mathrm{Si}$ waveguide dispersion for the above short period, with $\partial \mathrm{FSR}_{\mathrm{L}} / \partial \omega>0$, we thus increase the period $a$ to reduce the dispersive effect of the second term. We diminish the Littrow angle given by $\tan \theta_{L}=\mathrm{k}_{\mathrm{y}} / \mathrm{k}_{x}=w /(p a)$. We are, thus, closer to the FP dispersion. The total dispersion is then found as above, by solving for $\omega_{\mathrm{p}}$ with $n_{\mathrm{z}}=n_{\mathrm{z}}\left(\omega_{\mathrm{p}}\right)$, then forming FSR $\mathrm{p}_{\mathrm{p}}$ and here eventually the FSR dispersion $\Delta_{\mathrm{p}} \equiv \mathrm{FSR}_{\mathrm{p}+1}-\mathrm{FSR}_{\mathrm{p}}=\nu_{\mathrm{p}+1}+\nu_{\mathrm{p}-1}-2 \nu_{\mathrm{p}}$ $=\left(\omega_{\mathrm{p}+1}+\omega_{\mathrm{p}-1}-2 \omega_{\mathrm{p}}\right) / 2 \pi$, whose zeros are tracked. In the same spirit, we consider symmetric silicon slabs with symmetric clad, as occurs when embedding a SoI chip with deposited silica. We use standard database for chromatic dispersion of $\mathrm{Si}$ and silica, ${ }^{21}$ targeting a wavelength range $\lambda=1.4 \mu \mathrm{m}$ to $4.0 \mu \mathrm{m}$. As waveguide dispersion matters, we study seven different silicon thicknesses $d$ from $d=200 \mathrm{~nm}$ to $600 \mathrm{~nm}$, the latter being still monomode for the larger wavelengths of interest. We first plot on Fig. 3 the resulting frequencies of near-zero dispersion as a function of lattice period $a$. We also superimpose the corresponding constant-Littrow-angle loci for selected angles form $10^{\circ}$ to $24^{\circ}$ as dashed lines. The inset depicts the FSR differences $\Delta_{\mathrm{p}}$ vs. frequency for the case $(d=200 \mathrm{~nm}, a \sim 1 \mu \mathrm{m}$, $\nu \sim 150 \mathrm{THz}$, and $\theta_{\mathrm{L}} \sim 24^{\circ}$ ) pointed by a black arrow together with the similar quantity for a bulk silicon resonator and a principle FP carved in a raw waveguide. Exact values at which $\Delta_{\mathrm{p}}$ crosses zero are asymptotically independent of size $w$ : A large $w$ simply lessens discretization. For instance, for $d=200 \mathrm{~nm}$, $\mathrm{L}=133.6 \mu \mathrm{m}$ corresponds to an order $\mathrm{p}=500$ of the FP resonator with $n_{\mathrm{z}}=2.807$ at $\lambda=1500 \mathrm{~nm}$. Such a large order limits the typical excursions of $\Delta_{\mathrm{p}}$ to $10-100 \mathrm{MHz}$.

It appears from this extended scan of a basic siliconbased system with only periodicity as a parameter that the choice of the zero-dispersion frequency can readily cover a broad range of mid-IR to near-IR frequencies, associated to wavelengths $1.4-4.0 \mu \mathrm{m}$. This study of the simplest generalization of Littrow resonators thus reveals favourable perspectives for the realisation of resonators without dispersion, crucial to frequency combs.

Compared to previous schemes, the main requirement is not a precise dimension per se, but rather the achievement of

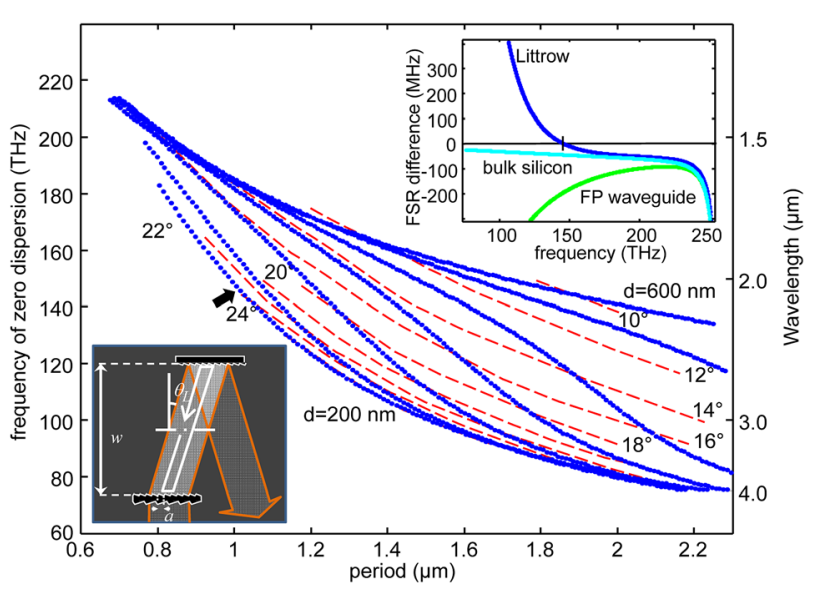

FIG. 3. Plot of the frequencies of zero dispersion (dots merging to solid lines) for initial silicon waveguide slabs of different thicknesses (from bottom to top, $d=200,240,300,360,420,500$, and $600 \mathrm{~nm}$ ), as a function of the Littrow grating period $a$. The assumption is here the band edge one, $k_{x}=\pi / a$ for the resonances cf. Fig. 1(b), which can be made by feeding the resonator through a double period grating on a mirror (bottom left inset); The dotted lines represent the Littrow angle at the zero dispersion situation thus realised, labelled from $10^{\circ}$ to $24^{\circ}$ by $2^{\circ}$ steps. The top right inset represents the dispersion of resonators made in bulk silicon (middle line), in a $200 \mathrm{~nm}$ silicon slab, and that of the Littrow resonator $(\mathrm{d}=200 \mathrm{~nm}, a \sim 1 \mu \mathrm{m}$, $\nu \sim 150 \mathrm{THz}$, and $\theta_{\mathrm{L}} \sim 24^{\circ}$ ). 
sufficiently high Littrow-type diffraction efficiency on a broad band, to rival with the high finesses of microrings, microtores, and microspheres. Progresses in integrated grating diffraction efficiency are tackled in spectrometer devices. ${ }^{22}$ The hope to operate at finesses around $500-1000$ is substantiated by the so-called "high-index contrast grating" (HCG) made of highindex suspended rods, which are made to operate in the demanding vertical cavity surface-emitting laser (VCSEL) devices since a decade. ${ }^{23}$ Adapting similar strategies to our in-plane blazed angle demands could offer equally high efficiencies close to $99.5 \%$ (in addition, note that the normal incidence channel in Fig. 3 inset should not be considered as a loss). Pure silicon gratings have also been made adequate for very specific highly resonant cavities where ultimate stability was sought. ${ }^{24}$ Another option is to recourse to some flavour of distributed feedback that is known to limit vertical scattering by delocalizing the reflection (at the expense of an extra dispersion to be included and mastered, though). This option has been worked out for spectrometers. ${ }^{25}$

In conclusion, we exhibit a first demonstration of strong anomalous dispersion and several handles to understand realistic silicon-based resonators. Though, it is clear that a lot of work remains to be done to practically operate "dispersion liberated" resonators of this kind. Issues are for instance, understanding whether simpler blazed gratings could help reaching a high Q; if their phase would then add a favourable imprint on the "bare" Littrow dispersion calculated here; the role of the device access guides. We also acknowledge that the possible frequency combs that would stem from such devices would have to be further engineered for expanding the zerodispersion range and favouring the "mode alignment" due to nonlinear mechanisms to be detailed yet. Nevertheless, we find it promising that our proposed silicon-based designs offer sufficient flexibility to get dispersion compensation with still a lot of freedom in FSR value, in compensation wavelength and in higher-order dispersion, to cite a few key quantities for the generation of frequency combs.
${ }^{1}$ Y. K. Chembo and N. Yu, Phys. Rev. A 82(3), 033801 (2010).

${ }^{2}$ V. S. Ilchenko, A. A. Savchenkov, A. B. Matsko, and L. Maleki, J. Opt. Soc. Am. A 20(1), 157 (2003).

${ }^{3}$ I. H. Agha, Y. Okawachi, M. A. Foster, J. E. Sharping, and A. L. Gaeta, in Nonlinear Photonics (2007), Vol. OSA Technical Digest, paper NTuC1.

${ }^{4}$ A. A. Savchenkov, A. B. Matsko, W. Liang, V. S. Ilchenko, D. Seidel, and L. Maleki, Nat. Photonics 5(5), 293 (2011).

${ }^{5}$ M. A. Foster, J. S. Levy, O. Kuzucu, K. Saha, M. Lipson, and A. L. Gaeta, Opt. Express 19(15), 14233 (2011).

${ }^{6}$ Y. Okawachi, K. Saha, J. S. Levy, H. Wen, M. Lipson, and A. L. Gaeta, Opt. Lett. 36(17), 3398 (2011).

${ }^{7}$ P. Del'Haye, T. Herr, E. Gavartin, M. L. Gorodestky, R. Holzwarth, and T. J. Kippenberg, Phys. Rev. Lett. 107(6), 063901 (2011).

${ }^{8}$ Q. Liu, S. Gao, L. Cao, and S. He, J. Opt. Soc. Am. B 29(2), 215 (2012).

${ }^{9}$ D. Zhu, Y. Zhou, X. Yu, P. Shum, and F. Luan, Opt. Express 20(24), 26285 (2012).

${ }^{10}$ O. Khayam, C. Cambournac, H. Benisty, M. Ayre, H. Brenot, G. H. Duan, and W. Pernice, Appl. Phys. Lett. 91(4), 041111 (2007).

${ }^{11}$ H. Kurt, H. Benisty, T. Melo, O. Khayam, and C. Cambournac, J. Opt. Soc. Am. B 25(12), C1 (2008).

${ }^{12}$ O. Khayam, H. Benisty, and C. Cambournac, Phys. Rev. B 78(15), 153107 (2008).

${ }^{13}$ H. Benisty, Photonics Nanostr. Fundam. Appl. 7, 115 (2009).

${ }^{14}$ O. Khayam and H. Benisty, Opt. Express 17(17), 14634 (2009).

${ }^{15}$ H. Benisty, N. Piskunov, P. N. Kashkarov, and O. Khayam, Phys. Rev. A 84, 063825 (2011).

${ }^{16}$ L. G. Velychko and Y. K. Sirenko, Prog. Electromagn. Res. B 16(1), 85 (2009).

${ }^{17}$ P. Dumon, W. Bogaerts, R. Baets, J. M. Fedeli, and L. Fulbert, Electron. Lett. 45(12), 581 (2009).

${ }^{18}$ S.-H. Baik, S.-K. Park, C.-J. Kim, and B. Cha, Opt. Laser Technol. 39, 262 (2007).

${ }^{19}$ W. H. P. Pernice, F. P. Payne, and D. F. G. Gallagher, Eur. Phys. Lett. D 82, 54001 (2008).

${ }^{20}$ H. Benisty, O. Khayam, and C. Cambournac, Photonics Nanostr. Fundam. Appl. 8, 210 (2010).

${ }^{21}$ M. Bass, Handbook of Optics, 3rd ed. (McGraw-Hill, 2009).

${ }^{22}$ P. J. Bock, P. Cheben, J. H. Schmid, A. V. Velasco, A. Delâge, S. Janz, D.-X. Xu, J. Lapointe, T. J. Hall, and M. L. Calvo, Opt. Express 20(18), 19882 (2012).

${ }^{23}$ C. J. Chang-Hasnain, Semicond. Sci. Technol. 26(1), 014043 (2011).

${ }^{24}$ F. Brückner, D. Friedrich, T. Clausnitzer, M. Britzger, O. Burmeister, K. Danzmann, E.-B. Kley, A. Tünnermann, and R. Schnabel, Phys. Rev. Lett. 104(16), 163903 (2010).

${ }^{25}$ P. Pottier and M. Packirisamy, IEEE J. Lightwave Technol. 30(17), 2922 (2012). 